Proceedings

\title{
Emerging and Legacy Organic Halogenated Pollutants in Indoor Dusts ${ }^{\dagger}$
}

\author{
Giulia Simonetti ${ }^{1}$, Elisa Sonego ${ }^{1, *}$, Federica Castellani ${ }^{~}$, Patrizia Di Filippo ${ }^{2}$, Carmela Riccardi ${ }^{2}$, \\ Donatella Pomata ${ }^{2}$ and Francesca Buiarelli ${ }^{1}$ \\ 1 Department of Chemistry, Sapienza University of Rome, Piazzale Aldo Moro, 5, 00185 Rome, Italy; \\ giulia.simonetti@uniroma1.it (G.S.); federica.castellani@uniroma1.it (F.C.); \\ francesca.buiarelli@uniroma1.it (F.B.) \\ 2 DIT, INAIL, Via Roberto Ferruzzi 38, 00143 Rome, Italy; p.difilippo@inail.it (P.D.F.); \\ ca.riccardi@inail.it (C.R.); d.pomata@inail.it (D.P.) \\ * Correspondence: elisa.sonego@uniroma1.it \\ + Presented at the 2st International Electronic Conference on Environmental Health Sciences, \\ 4-29 November 2019; Available online: https://iecehs-2.sciforum.net/.
}

Published: 5 November 2019

\begin{abstract}
In indoor environments, the concentration of halogenated organic pollutants in dust can be high due to the presence of products treated with these chemicals. In this study, we monitored emerging organic pollutants, such as novel brominated flame retardants (nBFRs) and some perfluoroalkyl substances (PFAS), together with legacy polychlorinated biphenyls (PCB) and polybrominated diphenyl ethers (PBDEs) in settled dust collected in five different (domestic and occupational) environments. In workplaces, a high incidence of PCBs, PBDEs and nBFRs occurred. Dust collection represents a simple, fast and cost-effective sampling and dust contamination level can be a useful indicator of environment healthiness.
\end{abstract}

Keywords: multiclass; priority; settled dust; environment monitoring; preventive measure

\section{Introduction}

In indoor environments such as houses and workplaces, the concentration of organic pollutants in dust can be high, due to the presence of textile, electronic, building materials and furniture treated with these chemicals. Among them, we focused on different classes of halogenated compounds, mostly used as flame retardants (FRs), such as novel brominated flame retardants (nBFRs) and some perfluoroalkyl substances (PFAS), together with legacy polychlorinated biphenyls (PCBs) and polybrominated diphenyl ethers (PBDEs). PCBs were used in thousands of consumer products. Some of them, called dioxin-like, have chemical-physical characteristics that make them similar to dioxins and furans, causing therefore the same toxicological effects. In addition, PBDEs, due to structural similarity with thyroxine, the main thyroid hormone, may interfere with the endocrine system. The association between high concentrations of some PBDEs in adipose tissues and the onset of cancer was also found. Another potential adverse effect has been shown in the neurological development of exposed children [1].

Due to the ban $[2,3]$ on using the aforementioned PCBs and PBDEs, but owing to their essential role, new brominated flame retardants (nBFRs) have been placed on the market. For these alternative chemicals, little or no toxicity data exist, therefore there is no scientific evidence that these products are safer than the banned ones. Hence, these compounds are of health concern because it would be expected behaviour similar to legacy ones, such as bioaccumulative and toxicity properties, including endocrine toxicity and carcinogenicity. Among them, tetrabromobisphenol A (TBBPA) is the most 
highly produced brominated flame retardant in the world, broadly added to printed circuit boards and to several types of polymers. Nevertheless, an environmental risk was identified and legislative restrictions were applied in Europe through the IPPC (Integrated Pollution Prevention and Control) Directive, since 1995 [4]. To date, effects on reproductive and nervous system development, including brain and thyroid function were recorded. TBECH is used in construction materials, electric cables, polystyrene-based insulation panels, plastics and adhesives. In vitro studies have shown the ability to bind and activate the androgen receptor (hAR) with high affinity. Even low-level exposure may cause behavioural, functional and developmental disorders [5].

Perfluorinated alkylated substances (PFAS) are analogously added to many materials to increase their resistance. In particular, perfluoroctane acid (PFOA) and perfluoroctane sulfonic acid (PFOS) were extensively produced and used. Many in vivo and in vitro studies have suggested that exposure to PFOS may lead to adverse effects on human health, such as hepatotoxicity, neurotoxicity, reproductive toxicity, immunotoxicity, thyroid disruption, cardiovascular toxicity, pulmonary toxicity, and renal toxicity [6-8].

Electronic and electric components, insulation wires, cables, and computers, televisions, electronic equipment, small and large domestic appliances, furniture, upholstery, mattresses, carpets and furniture can be sources of the above-mentioned compounds in indoor environments such as houses, offices, and mechanical and electronic workshops. Especially during processing of old and new devices and furniture, particles containing legacy and emerging halogenated pollutants can be released in the environment [9]. Hence, a simple, fast and cost-effective chemical characterization of indoor dust can be a useful indicator of environmental unhealthiness.

In this study, we monitored selected emerging organic pollutants, 13 nBFRs and 5 PFAS, together with 21 PCBs and 12 PBDEs in settled dusts in different indoor environments. Both domestic and workplace dusts were analysed, in order to investigate where the potential major exposure occurs.

\section{Methods}

The dust samples were collected during Summer 2019 in five different sampling sites; two domestic environments (D1, D2), an office (D3), in an electronic workshop (D4) and in a mechanical (D5) one, by using a household vacuum cleaner. Starting from our previous works, which focused on FRs occurrence in air and dust of an electrical and electronic waste treatment facility [10,11], we improved the analytical method to extend the investigation to new compounds. After sampling, dusts were sieved at $63 \mu \mathrm{m}$ and extracted by accelerated solvent extraction. The chromatographic mass spectrometric analyses were performed with both reversed phase high performance liquid chromatography (RP-HPLC) and gas chromatography (GC) equipped with a methylpheyl (5\%) silicone column. In particular an HPLC 1260 Infinity II system (Agilent Technologies Italia S.p.A., Cernusco sul Naviglio MI, Italy), coupled to a triple quadrupole mass spectrometer API 2000 (AB SCIEX S.r.l. Forster City, CA, USA) was used. HPLC-MS/MS was fitted with both ESI (electrospray ionization) and APCI (atmospheric pressure chemical ionization) sources. For GC-MS analyses, an HP 7890 B gas chromatograph fitted with an HP 5977B single quadrupole mass-selective detector (Agilent Technologies, Palo Alto, CA) was used in negative chemical ionization (NCI).

Determination of method performances and quantitative analyses were carried out as described in Buiarelli et al. [10].

\section{Results and Discussion}

Thanks to the optimized sample preparation for the analytical determination of this broad and heterogeneous group of compounds, we obtained extraction recoveries of about $88 \%$.

After the analyses of D1, D2, D3, D4 and D5 dusts, the results highlighted that in the electronic and mechanical workshops (D4 and D5), besides a high incidence of PCBs, PBDEs and nBFRs, PFOA was also detected. By contrast, the total contribution of the investigated pollutants was lower in house (D1 and D2) and in office (D3) dusts except for few nBFRs. 
The total contamination obtained by summing the contribution of each analyte showed the following pattern: D4 > D5 > D2 > D3 > D1 (data not shown); hence, D4 and D5 were the most contaminated environments.

For each sampling site, the sum of the compound concentration belonging to the same class $(\Sigma$ PCBs, $\Sigma$ PBDEs and $\Sigma$ nBFRs), was calculated and reported in the histograms of Figure 1.

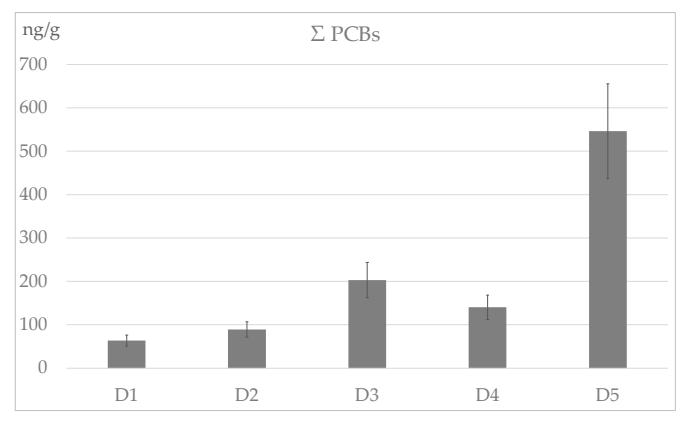

(a)

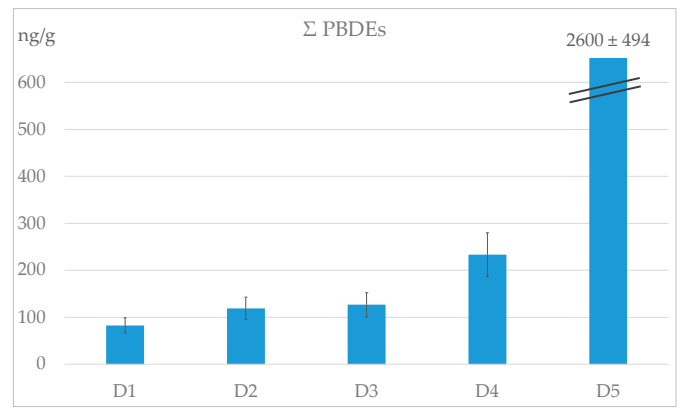

(b)

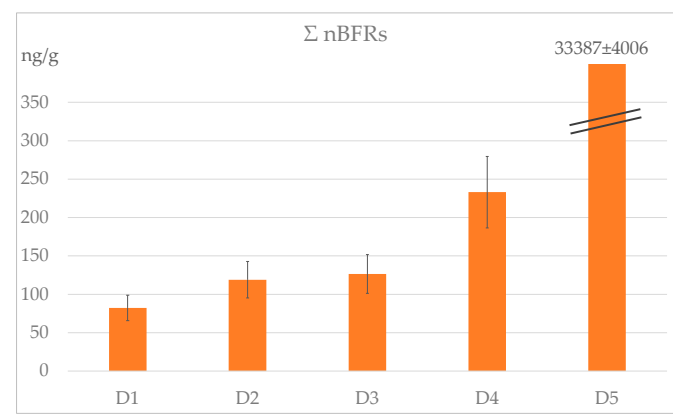

(c)

Figure 1. $\Sigma$ polychlorinated biphenyls (PCBs) (a), $\Sigma$ polybrominated diphenyl ethers (PBDEs) (b), and $\Sigma$ novel brominated flame retardants (nBFRs) (c) in $\mathrm{ng} / \mathrm{g}$ detected in each dust sample.

The higher concentration of $\Sigma$ PCBs (Figure 1a) was found in workplace dusts in the following order: D5 > D3 > D4, with D5 value about four times higher than D4, due to the larger size and the old age of D5 workplace, where there is a prevalence of a great number of old electronic devices [12].

In absence of a specific limit $\Sigma$ PCBs in dust, we compared the obtained concentrations with the value related to the soil, presented in regional screening level tables calculated from EPA (Environmental Protection Agency) to prevent carcinogenic risk resulting from long term exposure to PCBs [13], equal to $0.22 \mu \mathrm{g} / \mathrm{g}$. In D5, the PCB concentration of $0.55 \mu \mathrm{g} / \mathrm{g}$ exceeded more than twice the EPA value, proving to be the only alarming result. Furthermore, we monitored eight dioxin-like PCBs (DL PCBs), due to the health concern about these substances. The $\Sigma$ DL PCBs was calculated, and their percentage versus $\Sigma$ PCBs was above $50 \%$ for all samples except for D4.

Our findings about $\Sigma$ PBDEs are summarized in Figure $1 \mathrm{~b}$. The $\Sigma$ PBDEs was higher in D4 and D5, moreover D4 resulted more than twice of D1, D2 or D3. It is noteworthy that the $\Sigma$ PBDE value in D5, strongly affected by the PBDE 209, was detected in the highest concentration among the PBDEs. PBDE 209 is the most widespread PBDE, found in high concentration in several studies [14-16].

The $\Sigma$ nBFRs was reported in Figure 1c; in this study, we investigated 13 nBFRs, including TBBPA, which was the most concentrated pollutant detected. The D4 contamination was characterized by the prevalence of TBBPA, with a concentration of $32,320 \mathrm{ng} / \mathrm{g}$, perhaps due to the worker practise to remove the dust stored inside the devices before repairing them. High TBBPA concentrations were in agreement with other studies carried out in similar workplaces [17], as expected for its widespread use instead of octaBDE formulation [18]. Except D4, the $\Sigma$ nBFRs was comparable in all sampling sites, with a high value detected in a domestic dust (D2). 
Regarding the five PFAS investigated in this study, PFOA was only detected with a concentration less than LOQ (limit of quantification) $(119 \mathrm{ng} / \mathrm{g})$ in both D4 and D5 samples, whilst PFBS (perfluorobutane sulfonate), PFOS, PFTeDA (perfluorotetradecanoic acid) and FOSA (perfluorooctanesulfonamide) were not detected in any samples. Because of the PFOS and PFOA phase out, analysis of other PFAS, used alternatively, will be the next step in our scientific investigation.

In order to compare the contamination profile in the different indoor environments, pie charts are shown in Figure 2. They show the percentage distribution for the $\Sigma$ PCBs; $\Sigma$ PBDEs and $\Sigma$ nBFRs at each site.

D1

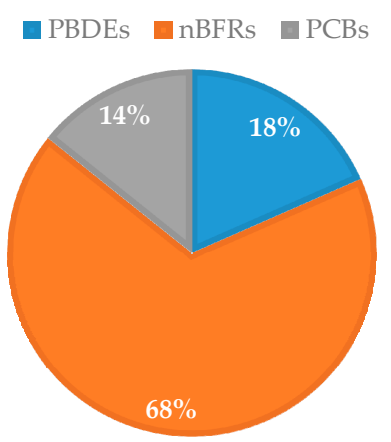

D3

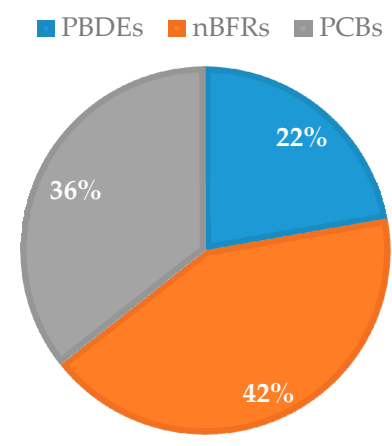

D4

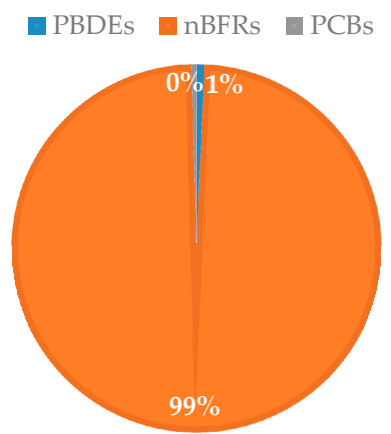

D2

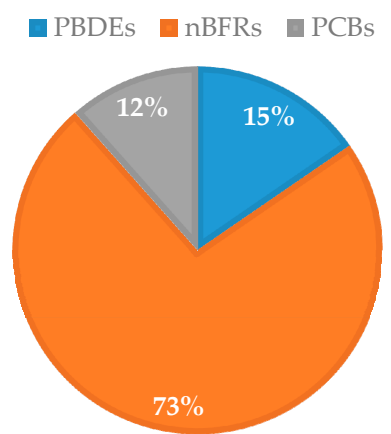

Figure 2. Percentage pie chart for the distribution of $\Sigma$ PCBs; $\Sigma$ PBDEs and $\Sigma$ nBFRs for each dust sample.

Domestic dust (D1 and D2) show a similar compound distribution, with a predominance of nBFRs. All pollutant categories contribute, almost in equal measure, to D3 and D5 contamination. The relative abundance of PCBs may be due to the presence of old electric and electronic devices and furniture. The relevant concentration of TBBPA in D4 yields a percentage of $99 \% \Sigma \mathrm{nBFR}, 1 \% \Sigma$ PCBs and $0 \% \Sigma$ PDBEs. However, the same percentage calculated without TBBPA concentration was slightly different, yielding $82 \% \Sigma \mathrm{nBFR}, 11 \% \Sigma$ PCBs and $7 \% \Sigma$ DBEs. The very peculiar concentration profile in D4 is due to the modern devices stored and repaired in the workplace. Finally, the results obtained in all sites highlight the importance to monitor nBFRs.

\section{Conclusions}

Since dust contamination level can be a useful indicator of environmental healthiness, we applied an analytical method for the determination of 51 legacy and emerging pollutants, belonging to different chemical classes, in indoor dust from life and work places. The mechanical and electronic workshops showed to be the more contaminated indoor environments, where high concentrations of PCBs, PBDEs and nBFRs occurred. The $\Sigma$ PCBs contamination in D5 resulted above the EPA Regional 
Screening Level to prevent carcinogenic risk. Moreover, TBBPA and PBDE 209, found in D4 and D5 respectively, were the most concentrated compounds detected in this study. The results suggest the need to still monitor the legacy PCBs and PBDEs, especially in workplaces where aged devices are handled, and the high occurrence of nBFRs due to the modern electronic equipment highlight the wide spread of these emerging contaminants. The results obtained can be a basis for exposure estimation and risk assessment studies.

Funding: This work was supported by INAIL/RicercaDiscrezionale/2016-2018/P3O3/UO2.

Conflicts of Interest: The authors declare no conflict of interest.

\section{References}

1. Taheran, M.; Komtchou, S.; Lonappan, L.; Naji, T.; Brar, S.K.; Cledon, M. Technology Environmental issues of Polybrominated Diphenyl Ethers. Crit. Rev. Environ. Sci. Technol. 2017, 47, 1107-1142.

2. UNEP. Listing of tetrabromodiphenyl ether and pentabromodiphenyl ether. In Proceedings of the Fourth Meeting of the Conference of the Parties to the Stockholm Convention, Geneva, Switzerland, 4-8 May 2009; SC-4/18.

3. UNEP. Listing of hexabromodiphenyl ether and heptabromodiphenyl ether. In Proceedings of the Fourth Meeting of the Conference of the Parties to the Stockholm Convention, Geneva, Switzerland, 4-8 May 2009; SC-4/14.

4. International Programme on Chemical Safety. Environmental Health Criteria 172. Tetrabromobisphenol A and Derivatives; World Health Organization: Geneva, Switzerland, 1995.

5. Khalaf, H.; Larsson, A.; Berg, H.; McCrindle, R.; Arsenault, G.; Olsson, P.-E. Diastereomers of the Brominated Flame Retardant 1,2-Dibromo-4-(1,2 dibromoethyl)cyclohexane Induce Androgen Receptor Activation in the HepG2 Hepatocellular Carcinoma Cell Line and the LNCaP Prostate Cancer Cell Line. Environ. Health Perspect. 2009, 117, 1853-1859.

6. Melzer, D.; Rice, N.; Depledge, M.H.; Henley, W.E.; Galloway, T.S. Association between Serum Perfluorooctanoic Acid (PFOA) and Thyroid Disease in the U.S. National Health and Nutrition Examination Survey. Environ. Health Perspect. 2010, 118, 686-692.

7. Darrow, L.A.; Groth, A.C.; Winquist, A.; Shin, H.-M.; Bartell, S.; Steenland, K. Modeled Perfluorooctanoic Acid (PFOA) Exposure and Liver Function in a Mid-Ohio Valley Community. Environ. Health Perspect. 2016, 124, 1227-1233.

8. Fitz-Simon, N.; Fletcher, T.; Luster, M.I.; Steenland, K.; Calafat, A.M.; Kato, K.; Armstrong, B. Reductions in serum lipids with a 4-year decline in serum perfluorooctanoic acid and perfluorooctanesulfonic acid. Epidemiology 2013, 24, 569-576.

9. Stubbings, W.A.; Harrad, S. Extent and mechanisms of brominated $\mathrm{fl}$ ame retardant emissions from waste soft furnishings and fabrics: A critical review. Environ. Int. 2014, 71, 164-175.

10. Buiarelli, F.; Di Filippo, P.; Pomata, D.; Riccardi, C.; Bartocci, M. A liquid chromatography tandem mass spectrometry method for simultaneous analysis of 46 atmospheric particulate-phase persistent organic pollutants and comparison with gas chromatography/mass spectrometry. Int. J. Environ. Anal. Chem. 2017, $97,1-22$.

11. Pomata, D.; Di Filippo, P.; Riccardi, C.; Rossi, V.; Simonetti, G.; Sonego, E.; Buiarelli, F. Method optimisation for the simultaneous determination of legacy and emerging halogenated flame retardants in particulate matter collected in an electronic waste recycling facility. Int. J. Environ. Anal. Chem. 2019. doi:10.1080/03067319.2019.1655007.

12. Iwegbue, C.M.A.; Eyengho, S.B.; Egobueze, F.E.; Odali, E.W.; Tesi, G.O.; Nwajei, G.E.; Martincigh, B.S. Polybrominated diphenyl ethers and polychlorinated biphenyls in indoor dust from electronic repair workshops in southern Nigeria: Implications for onsite human exposure. Sci. Total Environ. 2019, 671, 914927.

13. Rudel, R.A.; Seryak, L.M.; Brody, J.G. PCB-containing wood floor finish is a likely source of elevated PCBs in residents' blood, household air and dust: A case study of exposure. Environ. Health 2008, 7, 2.

14. Wang, J.; Wang, Y.; Shi, Z.; Zhou, X.; Sun, Z. Legacy and novel brominated fl ame retardants in indoor dust from Beijing, China: Occurrence, human exposure assessment and evidence for PBDEs replacement. Sci. Total Environ. 2018, 618, 48-59. 
15. Venier, M.; Vojta, Š.; Be, J.; Romanak, K.; Melymuk, L.; Krátká, M.; Kuku, P.; Okeme, J.; Saini, A.; Diamond, M.L.; et al. Brominated flame retardants in the indoor environment-Comparative study of indoor contamination from three countries. Environ. Int. 2016, 94, 150-160.

16. Wemken, N.; Drage, D.S.; Abdallah, M.A.-E.; Harrad, S.; Coggins, M.A.; Coggins, A.M. Concentrations of Brominated Flame Retardants in Indoor Air and Dust from Ireland Reveal Elevated Exposure to Decabromodiphenyl Ethane. Environ. Sci. Technol. 2019, 53, 9826-9836.

17. Sjödin, A.; Carlsson, H.; Thuresson, K.; Sjölin, S.; Bergman, Å.; Östman, C. Flame retardants in indoor air at an electronics recycling plant and at other work environments. Environ. Sci. Technol. 2001, 35, 448-454.

18. Abafe, O.A.; Martincigh, B. Determination and human exposure assessment of polybrominated diphenyl ethers and tetrabromobisphenol A in indoor dust in South Africa. Environ. Sci. Pollut. Res. 2016, 23, 70387049 .

(C) 2019 by the authors. Licensee MDPI, Basel, Switzerland. This article is an open access article distributed under the terms and conditions of the Creative Commons Attribution (CC BY) license (http://creativecommons.org/licenses/by/4.0/). 